NASA/TP-2005-213941

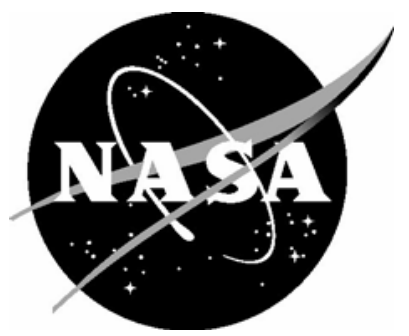

Numerical Study of the Generation of Linear Energy Transfer Spectra for Space Radiation Applications

Francis F. Badavi

Christopher Newport University, Newport News, Virginia

John W. Wilson

NASA Langley Research Center, Hampton, Virginia

Abigail Hunter

University of Utah, Salt Lake City, Utah 
Since its founding, NASA has been dedicated to the advancement of aeronautics and space science. The NASA Scientific and Technical Information (STI) Program Office plays a key part in helping NASA maintain this important role.

The NASA STI Program Office is operated by Langley Research Center, the lead center for NASA's scientific and technical information. The NASA STI Program Office provides access to the NASA STI Database, the largest collection of aeronautical and space science STI in the world. The Program Office is also NASA's institutional mechanism for disseminating the results of its research and development activities. These results are published by NASA in the NASA STI Report Series, which includes the following report types:

- TECHNICAL PUBLICATION. Reports of completed research or a major significant phase of research that present the results of NASA programs and include extensive data or theoretical analysis. Includes compilations of significant scientific and technical data and information deemed to be of continuing reference value. NASA counterpart of peerreviewed formal professional papers, but having less stringent limitations on manuscript length and extent of graphic presentations.

- TECHNICAL MEMORANDUM. Scientific and technical findings that are preliminary or of specialized interest, e.g., quick release reports, working papers, and bibliographies that contain minimal annotation. Does not contain extensive analysis.

- CONTRACTOR REPORT. Scientific and technical findings by NASA-sponsored contractors and grantees.
- CONFERENCE PUBLICATION. Collected papers from scientific and technical conferences, symposia, seminars, or other meetings sponsored or co-sponsored by NASA.

- SPECIAL PUBLICATION. Scientific, technical, or historical information from NASA programs, projects, and missions, often concerned with subjects having substantial public interest.

- TECHNICAL TRANSLATION. Englishlanguage translations of foreign scientific and technical material pertinent to NASA's mission.

Specialized services that complement the STI Program Office's diverse offerings include creating custom thesauri, building customized databases, organizing and publishing research results ... even providing videos.

For more information about the NASA STI Program Office, see the following:

- Access the NASA STI Program Home Page at http://www.sti.nasa.gov

- E-mail your question via the Internet to help@sti.nasa.gov

- Fax your question to the NASA STI Help Desk at (301) 621-0134

- Phone the NASA STI Help Desk at (301) 621-0390

- Write to: NASA STI Help Desk NASA Center for AeroSpace Information 7121 Standard Drive Hanover, MD 21076-1320 
NASA/TP-2005-213941

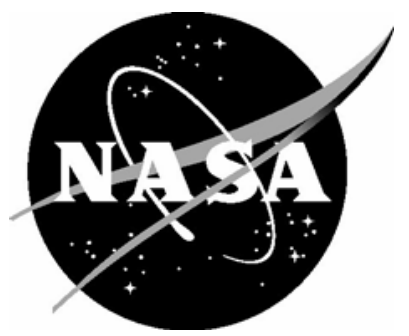

\section{Numerical Study of the Generation of Linear Energy Transfer Spectra for Space Radiation Applications}

Francis F. Badavi

Christopher Newport University, Newport News, Virginia

John W. Wilson

NASA Langley Research Center, Hampton, Virginia

Abigail Hunter

University of Utah, Salt Lake City, Utah

National Aeronautics and

Space Administration

Langley Research Center

Hampton, Virginia 23681-2199 
Available from:

NASA Center for AeroSpace Information (CASI)

7121 Standard Drive

Hanover, MD 21076-1320

(301) 621-0390
National Technical Information Service (NTIS) 5285 Port Royal Road Springfield, VA 22161-2171

(703) 605-6000 


\title{
Numerical Study of the Generation of Linear Energy Transfer Spectra for Space Radiation Applications
}

\author{
Francis F. Badavi \\ Christopher Newport University \\ Newport News, Virginia \\ John W. Wilson \\ NASA Langley Research Center \\ Hampton, Virginia \\ Abigail Hunter \\ University of Utah \\ Salt Lake City, Utah
}

\begin{abstract}
In analyzing charged particle spectra in space due to galactic cosmic rays (GCR) and solar particle events (SPE), the conversion of particle energy spectra into linear energy transfer (LET) distributions is a convenient guide in assessing biologically significant components of these spectra. The mapping of LET to energy is triple valued and can be defined only on open energy subintervals where the derivative of LET with respect to energy is not zero. Presented here is a well-defined numerical procedure which allows for the generation of LET spectra on the open energy subintervals that are integrable in spite of their singular nature. The efficiency and accuracy of the numerical procedures is demonstrated by providing examples of computed differential and integral LET spectra and their equilibrium components for historically large SPEs and 1977 solar minimum GCR environments. Due to the biological significance of tissue, all simulations are done with tissue as the target material.
\end{abstract}

\section{INTRODUCTION}

In accessing the biological response (risk) to radiation exposure, a customary assumption is that risk is related to the energy absorbed per unit mass within a macroscopic volume of the biological material. This implies a certain uniformity in the energy deposited to the exposed material, which in general is true for radiation of low linear energy transfer (LET) such as x-ray, $\gamma$-ray, and $\beta$-ray exposures. As experience from exposures with high LET radiation was gained, the non-uniformity of the energy deposited in the exposed material was clearly seen as an important determinant of biological response, and hence LET dependent relative biological effectiveness (RBE) factors came under intense study and provided the basis for the LET dependent quality factor (Q) for use in radiation protection (refs. 1-3). As a consequence of such exposure studies, the micro-dosimeter concept was developed as an instrument to measure such LET fluctuations.

Although the concept of an LET dependent RBE is useful for charged particles of relatively low kinetic energy, there still are serious concerns about the biological effects of high charge and energy (HZE) ions in deep space (outside the earth's protective geomagnetic field) resulting from the lateral spread of the energy deposited in 
the exposed material that is larger than a typical cell nucleus (ref. 4). In this case, LET is not sufficient to define an $\mathrm{RBE}$, but the track width also plays an important role in calculating the biological response. This was the origin of the limited LET concepts (refs. 1 and 5) as related to the lateral spread of the energy deposited in an exposed material. The importance of such track structure effects on biological response has been demonstrated for a number of biological systems (ref. 6).

Even though LET as a biological response indicator is most applicable to low charge and low energy particles, LET, due to its simplicity, is also considered a rough indicator for HZE particles as well. For this reason, we are interested in the generation of differential and integral LET distributions as a guide in identifying biologically significant components of HZE.

In this work, tissue due to its biological significance is used as the only target material. The methodology of differential and integral LET simulations as presented here are, however, applicable to any material such as silicon, the material of interest in microelectronics.

The transport of charged ions through tissue is numerically simulated using the deterministic ionizing radiation transport code HZETRN (High charge Z and Energy TRaNsport) developed at the Langley Research Center (LaRC). This code simulates the radiation shielding response of any material subject to exposure to three different environments, namely: the GCR environments in both low earth orbit (LEO) and free space, geomagnetically trapped proton fields, and historically large SPEs (ref. 7).

For GCR at LEO and free space, the environment of solar minimum (1977) is used. This epoch represents the worst case scenario (maximum spectral intensities) of the GCR environment. At LEO, the GCR environment is further modified (attenuated) by the effects of Earth's geomagnetic field. The international space station (ISS) trajectory (400 kilometer altitude, and 51.6 degrees inclination) is used to simulate the effect of GCR at LEO. The ISS case study is chosen here since recent data from the space shuttle flights (space transportation system, STS) to the high orbital inclination of ISS show that more than 50\% of accumulated dose incurred by ISS crew (except during SPE) are from high energy nuclei in the GCR spectra, and of that amount a substantial fraction is from highly charged and energetic heavy nuclei. The large contribution of these particles to the accumulated dose is due to the high orbital inclination of the ISS at 51.6 degree, since the geomagnetic field strength diminishes near the poles, and HZE particles that are deflected at lower latitudes are allowed to reach objects at high inclination LEO flights.

For SPE environments, parametric fits to the February 1956, November 1960, August 1972 (King and LaRC), September 29, 1989, and October 1989 events are used (ref. 7). For SPE simulations, only the free space environment is considered due to limited ability of SPE particles to penetrate the geomagnetic field except at very high latitudes.

The rest of this report is organized in the following manner: First, a somewhat detailed description of the mathematical and numerical modeling of differential LET spectra, its equilibrium component, and stopping power, with the connection between the two, as used in this paper is presented. This is followed by a description of the integral LET spectra and its equilibrium component. Next, to validate the efficiency of the numerical procedures in computing differential and integral LET spectra and their equilibrium components, the results of numerical simulations for a number of established SPEs and the 1977 solar minimum GCR environments in free space and LEO are presented. Finally, there is a brief concluding remarks section that includes suggestions for future work. 


\section{DIFFERENTIAL LET SPECTRA}

In radiobiology, relating biological response to the LET of the radiation environment is traditional. For example, RBE and the quality factor Q are generally taken as being related to LET. As a consequence, the concept of LET spectra has played a role in estimating biological response. Unfortunately, this concept is most useful if the flux $\left(\phi_{L}(L) d L\right)$ of particles with LET $(L)$ between $L$ and $L+d L$ is known. This is generally found by knowing the energy flux $\left(\phi_{E}(E) d E\right.$ ) of particles with energy $E$ between $E$ and $E+d E$, where $L$ is known as a function of $E$ so that $\phi(L)$ and $\phi(E)$ are related according to equation 1 below:

$$
\phi_{L}(L)=\left|\frac{d L}{d E}\right|^{-1} \phi_{E}(E)
$$

where the pre-factor $\left|\frac{d L}{d E}\right|^{-1}$ is the Jacobian between the $E$ and $L$ spaces. The difficulty with this approach is that $d L / d E=0$ at the maxima and minima of the LET curve and that $\phi_{L}(L)$ must be replaced by the sum over the various branch functions as:

$$
\phi_{L}(L)=\sum_{B}\left|\frac{d L}{d E}\right|_{B}^{-1} \phi_{E}\left(E_{B}\right)
$$

where $E_{B}$ is the energy of each branch associated with $L$. That is, for all values of $E_{B}$ the following must hold:

$$
L=L\left(E_{B}\right)
$$

Clearly, $\phi_{L}(L)$ does not exist for every value of $L$ but is defined on open intervals not containing values for which $d L / d E=0$. Furthermore, $\phi_{L}(L)$ is unbounded on the open subintervals over which it is defined, even though $\phi_{L}(L)$ is integrable over its domain. From the above arguments, enough challenges obviously exist in finding a representation for $\phi_{L}(L)$. This problem can be simplified since $L$ has but one maximum and one minimum other than at zero energy. Furthermore, $L$ in the neighborhood of the branch limits has continuous second derivative, allowing $L$ to have a Taylor series expansion (approximation) of the form:

$$
L \approx L\left(E_{B}\right)+\frac{1}{2} L^{\prime \prime}\left(E_{B}\right)\left(E-E_{B}\right)^{2}
$$

Combining equations 1 and 4, one can show that in the neighborhood of the branch limits $\phi_{L}(L)$ can be approximated by:

$$
\phi_{L}(L) \approx \phi_{E}\left(E_{B}\right)\left[\sqrt{2\left|L_{B}^{\prime \prime}\left(L-L_{B}\right)\right|}\right]^{-1}
$$

where the subscript $B$ denotes evaluation at the branch limit. 
The above considerations are implemented in the following manner. The LET is defined over a numerical grid given by the sequence $\left\{E_{i}\right\}$. The maximum and minimum branch points are found at $d L / d E=0$ and are noted by $E_{\max }$ and $E_{\min }$, respectively. The sequence $\left\{E_{i}\right\}_{L}$ is defined as those values of $E_{i}$ less than $E_{\max }$, with the main sequence $\left\{E_{i}\right\}_{m}$ being defined by $E_{\max }<E_{i}<E_{\min }$ and the sequence $\left\{E_{i}\right\}_{H}$ defined by $E_{\min }<E_{i}$. The three branch functions are then represented by:

$$
\left\{\phi_{L i}\right\}_{B}=\left\{\left|\frac{d E}{d L}\right|_{i} \phi_{E i}\right\} \quad E_{i} \in\left\{E_{i}\right\}_{B}
$$

where $B$ denotes one of the three branches (that is, $B=L, m$, or $H$ ). Having a table of values $\left\{\left(\phi_{L i}, L_{i}\right)\right\}_{B}$ in order to reconstruct an adequate representation of the function over each branch may not be sufficient because $\phi_{L i}$ is unbounded near the branch limits and an extrapolation into the neighborhood of the branch limit must be provided. This is accomplished by recognizing that at the branch point $\phi_{L}\left(L_{B}\right)$ can be approximated by:

$$
\phi_{L}\left(L_{B}\right) \approx{\sqrt{\left|L-L_{B}\right|}}^{-1}
$$

and if $\left\{E_{i}\right\}$ is sufficiently close to the branch point, equation 7a can be used to approximate the spectrum by:

$$
\phi_{L}(L) \approx \phi_{L i}(L) \phi_{L}\left(L_{B}\right) \sqrt{\left|L_{i}-L_{B}\right|}
$$

Combining equations $7 \mathrm{a}$ and $7 \mathrm{~b}$ imply that near the branch point $\phi_{L}(L)$ can be approximated by:

$$
\phi_{L}(L) \approx \phi_{L i}\left(\left|L_{i}-L_{B}\right| /\left|L-L_{B}\right|\right)^{1 / 2}
$$

where $L_{i}$ is the nearest grid value to the branch limit $L_{B}$ in the appropriate domain. Thus, the data set required to reconstruct the LET spectrum is the branch limit values of $E_{\text {max }}, E_{\min }, L_{\text {max }}$, and $L_{\text {min }}$ and the sequences $\left\{E_{i}\right\},\left\{L_{i}\right\}$, and $\left\{\phi_{E i}\right\}$. Note that the numerical values of the above parameters depend on the charge $\mathrm{Z}$ and mass $\mathrm{A}$

of the particles of the field. Thus, $E_{\max }, E_{\min }, L_{\max }$, and $L_{\min }$ must be specified for each ion type in the radiation field.

\section{STOPPING POWERS}

As stated earlier, the energy imparted to the medium by a passing charged particle is related to the stopping power (the energy loss per unit distance traveled in the medium) and is known as the linear energy transfer (LET), where $L=S(E)$. To calculate the spectrum of energy deposited, we must evaluate the LET spectrum given by equation 1.

Clearly, a method of generating $d(S(E)) / d(E)$ as a function of $E$ is required. As explained elsewhere (ref. $8)$, the stopping powers used in this work are taken from the fitted curves for protons and alpha particles of Ziegler 
at low energy (ref. 9), and are extrapolated using the Bethe formula at high energy. The stopping power of ions with a charge greater than 2 is scaled using the effective charge formalism of Barkas (ref. 10), and the corresponding alpha particle stopping power of Ziegler.

In the HZETRN code (ref. 7), the stopping power values are stored in a data array for 15 ion charge values ranging from 1 to 92 over an energy grid of 60 points between $0.01 \mathrm{MeV}$ and $50 \mathrm{GeV}$. Furthermore, extrapolation to lower energy or higher energy is accomplished by making the stopping power and its first derivative continuous at the boundaries of the energy grid. The numerical values are then scaled by $\ln \left[\frac{1}{Z^{2}} S(\ln E, Z)\right]$ to minimize numerical errors. The scaled data array is then fitted by a two-dimensional spline with appropriate boundary conditions from which $d(S(E)) / d(E)$ is found. Figure 1, below shows $S(E)$ and $d(S(E)) / d(E)$ as functions of energy $E$ for a target material of tissue composition for the projectile mass number in the range of 1 to 58 , with an energy range of $0.01 \mathrm{MeV}$ to $10 \mathrm{GeV}$.

S(E) vs. E for GCR lons in Tissue

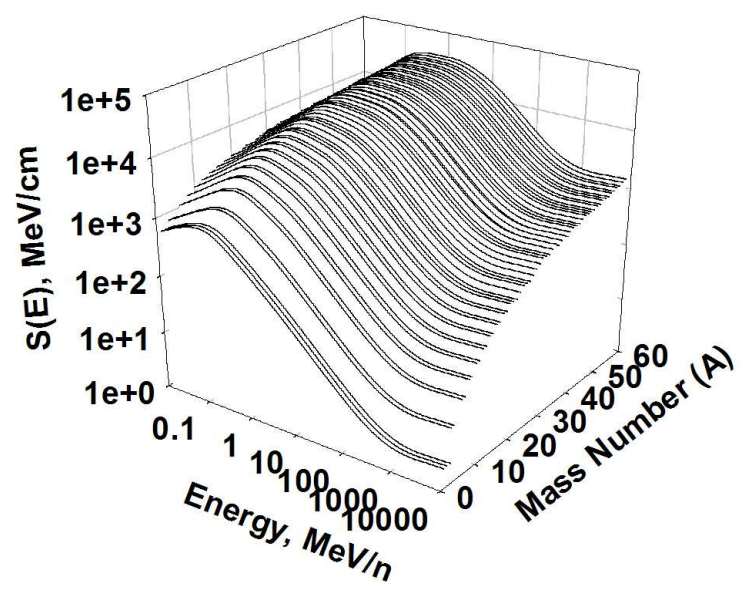

dS(E)/d(E) vs. E for GCR lons in Tissue

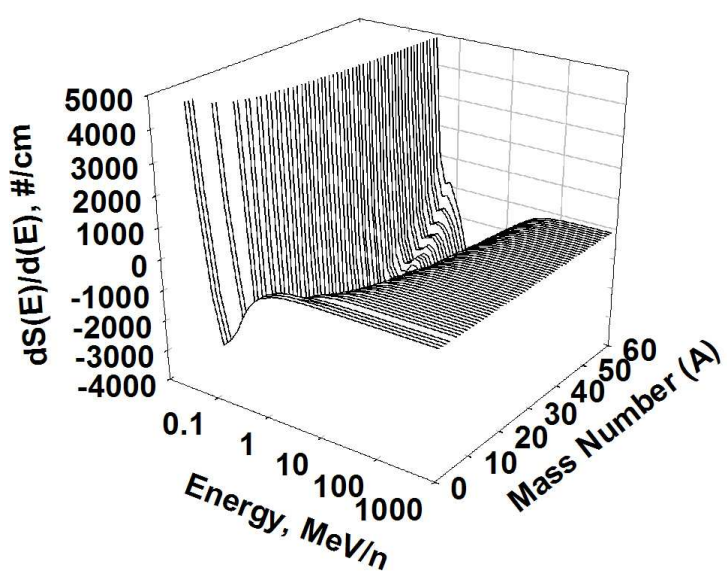

Figure 1. Stopping power and its derivative for ion range of 1-58 for a tissue composition.

The derivatives in the figure above vanish at $S(E)$ maximum at low energy where binding effects dominate and $S(E)$ minimum at high energy because of relativistic and density effects. The energies associated with the maximum and minimum $E_{\max }$ and $E_{\min }$ are shown in Figure 2, along with the corresponding values of $L_{\max }$ and $L_{\min }$. These provide the basic data for the construction of differential LET spectra. 
Energy values at branch limits

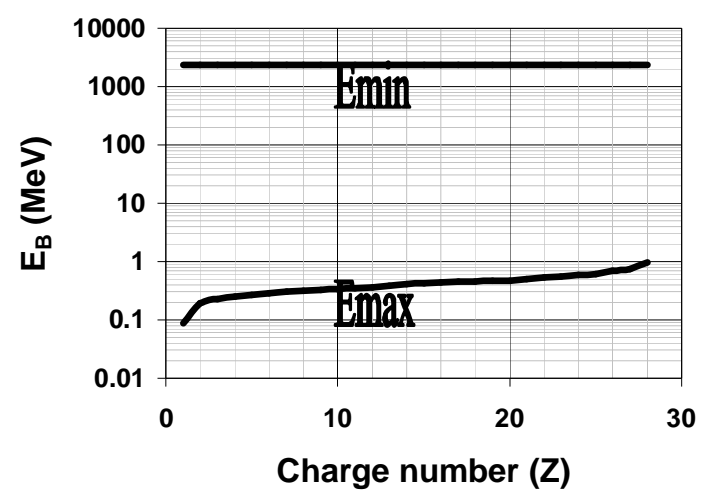

\section{LET values at branch limits}

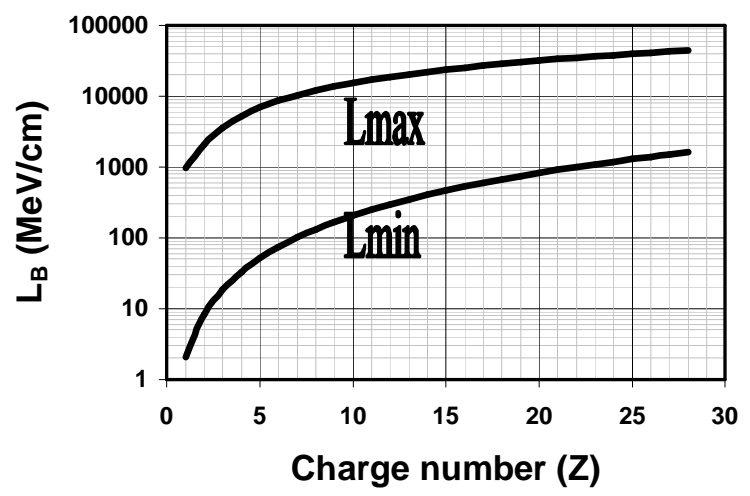

Figure 2. Values of energy and LET at branch points as a function of charge number Z.

\section{EQUILIBRIUM SPECTRA}

Regardless of whether the source of the projectile ions (GCR, SPE, etc...) is at the boundary or is an interior volume source, the accumulated fluence approaches a characteristic equilibrium spectrum that depends on the composition of the exposed medium. The equilibrium spectrum for volume sources has been given elsewhere (ref. 11). The fluence within a medium at a distance $x$ from the boundary is given by:

$$
\phi(x, E)=\frac{S\left(E_{x}\right)}{S(E)} \phi\left(0, E_{x}\right)
$$

where $\phi(0, E)$ is the fluence at the boundary, $E_{x}=R^{-1}[R(E)+x]$ is the energy at depth $x$, and $R(E)$ is the range of the individual particle with energy $E$ such that $E=R^{-1}[R(E)]$. The $\phi(x, E)$ term of equation (8) results from the formalism (refs. 11, 12) where the balance of high energy gains and low energy losses (ignoring the nuclear reactions) is given by:

$$
\left[\frac{\partial}{\partial x}-\frac{\partial}{\partial E} S(E)\right] \phi(x, E)=\text { Nuclear gains and losses }
$$

Excluding the nuclear processes, the solution of equation 9 can be expressed as:

$$
S(E) \phi(x, E)=S\left(E_{x}\right) \phi\left(0, E_{x}\right)
$$

It must be noted that when $x>R(E)$, then $E_{x} \approx R^{-1}(x)$ and a solution to equation (10a) is approximated by:

$$
\phi(x, E) \approx \frac{S\left[R^{-1}(x)\right] \phi\left[0, R^{-1}(x)\right]}{S(E)}
$$

which is the equilibrium spectral limit solution.

Figure 3, shows the differential LET spectra for the tissue thickness range of $0 \leq x \leq 50 \mathrm{~cm}$. for six of the historically larger SPEs of solar cycles 19 and 20, where as stated earlier the propagation through the tissue was calculated using the HZETRN code. The abundance of high energy protons in the February 1956 spectrum is evident with the low LET (high energy) singularity being seen in the figure. Furthermore, note that the existence of the higher energy spectra of the LaRC fit to the August 1972 event relative to the King spectrum is evident at the largest penetration depths. As is evident in Figure 3, the Bragg peak due to the protons is present at around 1000 $\mathrm{MeV} / \mathrm{cm}$ in all six spectrums, while the peak due to the helium secondary production at all depths beyond the boundary manifests itself at around $2000 \mathrm{MeV} / \mathrm{cm}$. 
Freespace dФ(L)/d(L) vs. L, for Feb.1956 SPE

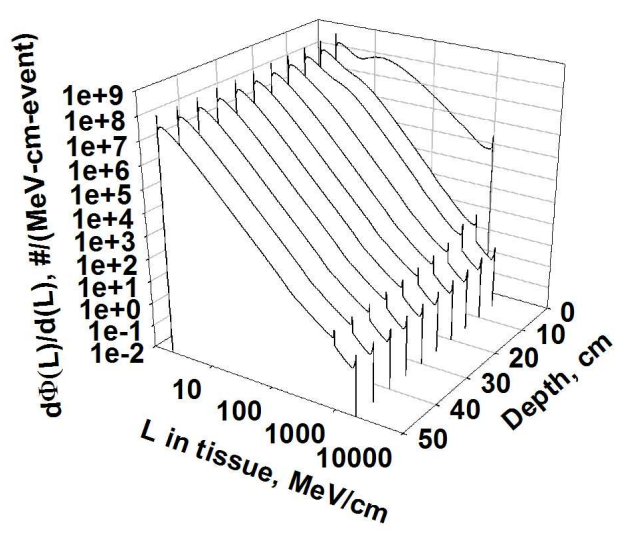

Freespace dФ(L)/dL vs. L for Aug. 1972 SPE (King)

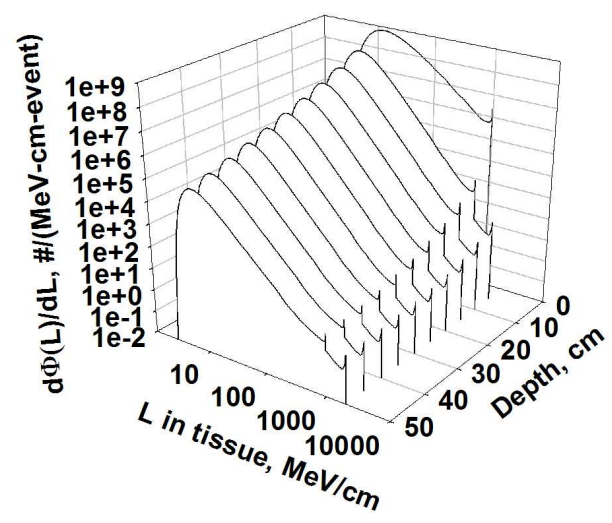

Freespace dФ(L)/dL vs. L for Sep. 1989 SPE

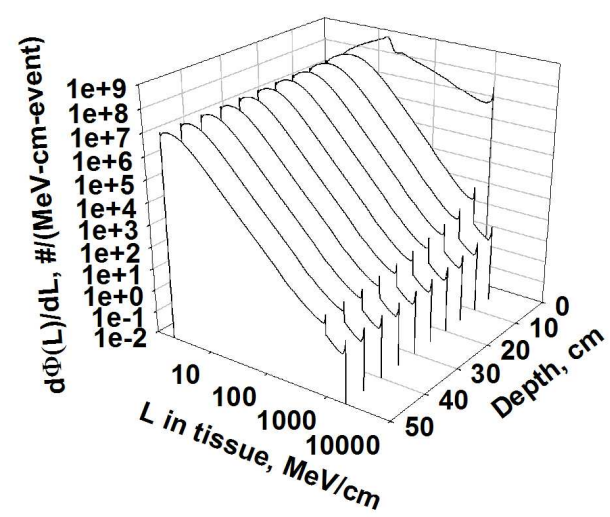

Freespace dФ(L)/d(L) vs. $L$ for Nov. 1960 SPE

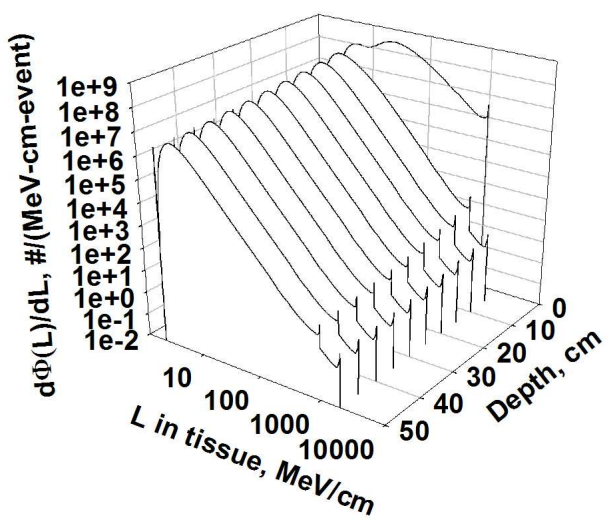

Freespace dФ(L)/dL vs. L for Aug. 1972 SPE (LaRC)

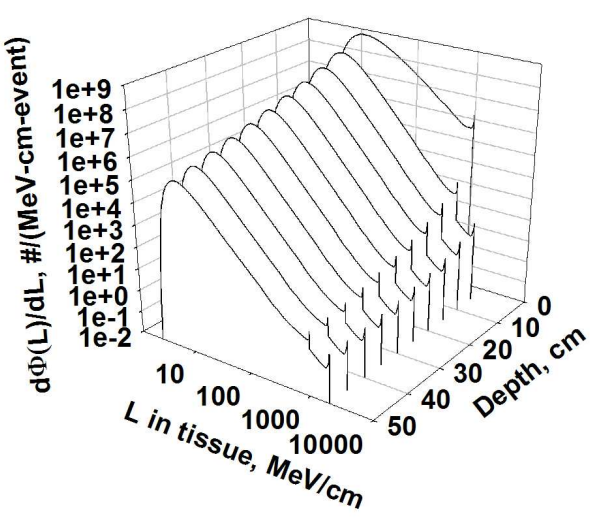

Freespace dФ(L)/d(L) vs. L for Oct. 1989 SPE

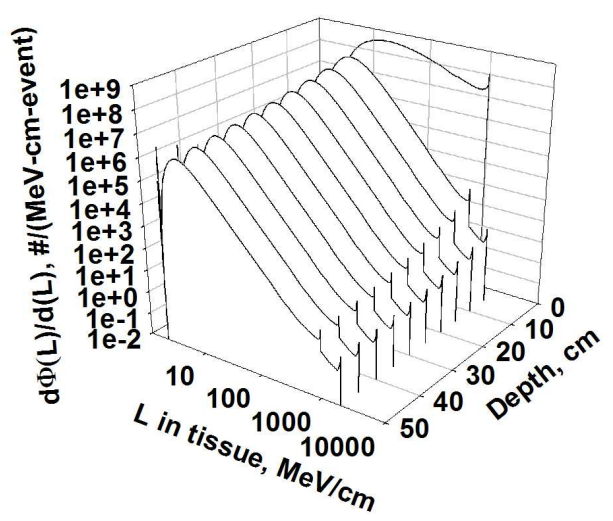

Figure 3. Differential LET spectra for six SPE events of cycle 19 and 20. 
The differential form of the fit for SPEs as represented by equations 11a.-11f. below, were used as the boundary condition to HZETRN to initiate propagation in tissue (ref. 7).

February 1956: $\quad \frac{d \phi_{P}(E)}{d(E)}=6.0 \times 10^{7} e^{-\left(\frac{E-10}{25}\right)}+9.375 \times 10^{5} e^{-\left(\frac{E-100}{320}\right)}$

November 1960: $\quad \frac{d \phi_{P}(E)}{d(E)}=6.33 \times 10^{8} e^{-\left(\frac{E-10}{12}\right)}+4.88 \times 10^{6} e^{-\left(\frac{E-100}{80}\right)}$

August $1972\left(\right.$ King): $\quad \frac{d \phi_{P}(E)}{d(E)}=2.98 \times 10^{8} e^{-\left(\frac{E-30}{26.5}\right)}$

August $1972(\operatorname{LaRC}): \quad \frac{d \phi_{P}(E)}{d(E)}=2.20 \times 10^{7} e^{-\left(\frac{E-100}{30}\right)}$

September 1989: $\quad \frac{d \phi_{P}(E)}{d(E)}=1.446 \times 10^{8}\left(\frac{E+938}{\sqrt{E(E+1876)}}\right) e^{-\left(\frac{\sqrt{E(E+1876)}}{102.118}\right)} \quad E<30 \mathrm{MeV}$

October 1989: $\quad \frac{d \phi_{P}(E)}{d(E)}=6.104 \times 10^{8}\left(\frac{E+938}{\sqrt{E(E+1876)}}\right) e^{-\left(\frac{\sqrt{E(E+1876)}}{92.469}\right)}$

The corresponding integral form of the fit for SPEs as measured by the ground or space based detectors are given by equations $12 \mathrm{a}-12 \mathrm{f}$. (ref. 7).

February 1956: $\quad \phi_{P}(>E)=1.5 \times 10^{9} e^{-\left(\frac{E-10}{25}\right)}+3 \times 10^{8} e^{-\left(\frac{E-100}{320}\right)}$

November 1960: $\quad \phi_{P}(>E)=7.6 \times 10^{9} e^{-\left(\frac{E-10}{12}\right)}+3.9 \times 10^{8} e^{-\left(\frac{E-100}{80}\right)}$

August $1972\left(\right.$ King): $\phi_{P}(>E)=7.9 \times 10^{9} e^{-\left(\frac{E-30}{26.5}\right)}$

August $1972(\mathrm{LaRC}): \phi_{P}(>E)=6.6 \times 10^{8} e^{-\left(\frac{E-100}{30}\right)}$

September 1989: $\quad \phi_{P}(>E)=1.477 \times 10^{10} e^{-\left(\frac{\sqrt{E(E+1876)}}{102.118}\right)}$ $E<30 \mathrm{MeV}$

$\phi_{P}(>E)=2.94 \times 10^{17}(\sqrt{E(E+1876)})^{-3.5}$

$E \geq 30 \mathrm{MeV}$

October 1989: $\quad \phi_{P}(>E)=5.644 \times 10^{10} e^{-\left(\frac{\sqrt{E(E+1876)}}{92.469}\right)}$ 
Figure 4. below is the graphical representation of the differential and integral forms of the six SPEs (equations 11 and 12 ) that are used as boundary conditions to HZETRN in this paper.

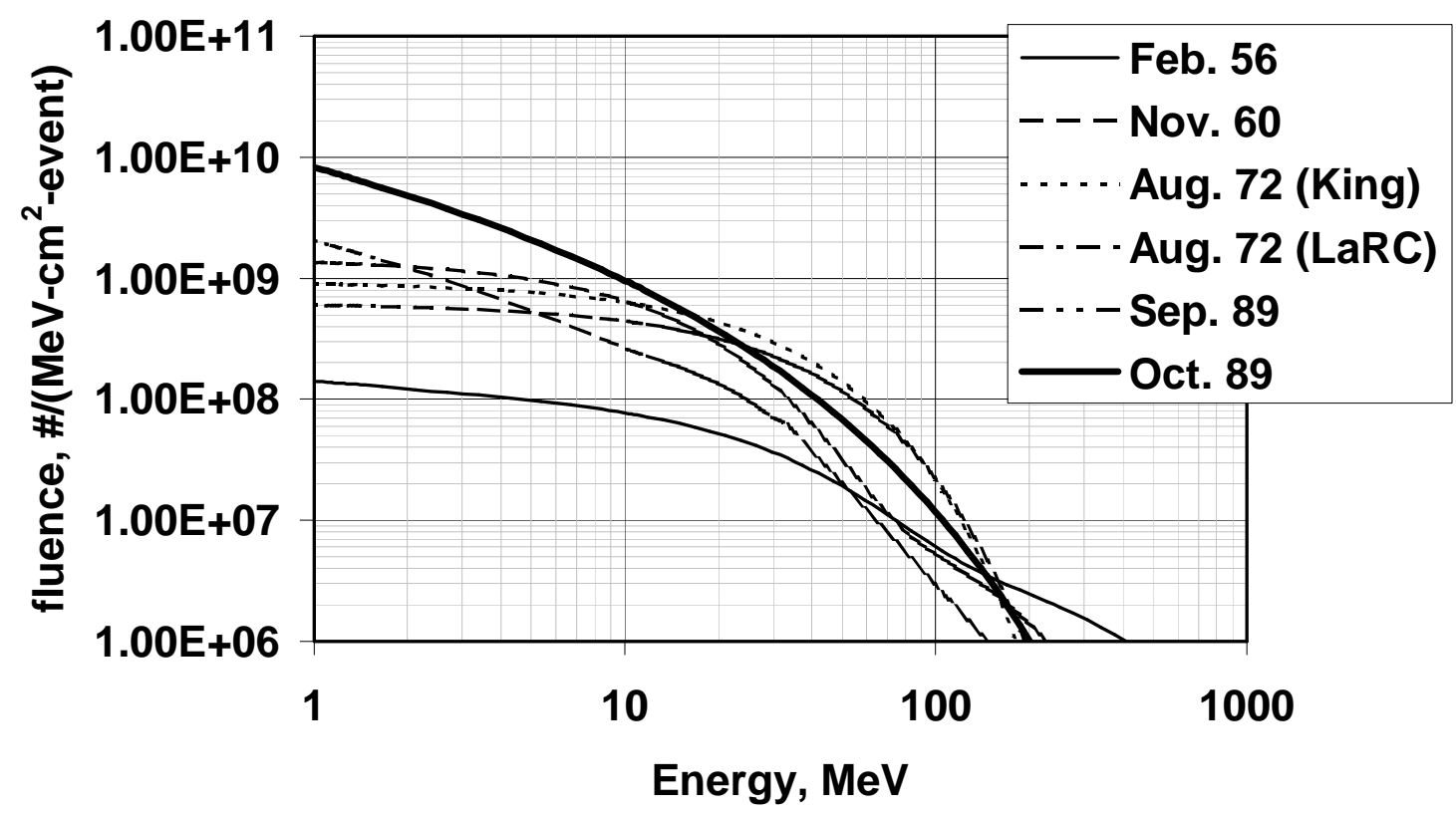

Figure 4a. Differential fluence spectra for six SPE events of cycle 19 and 20.

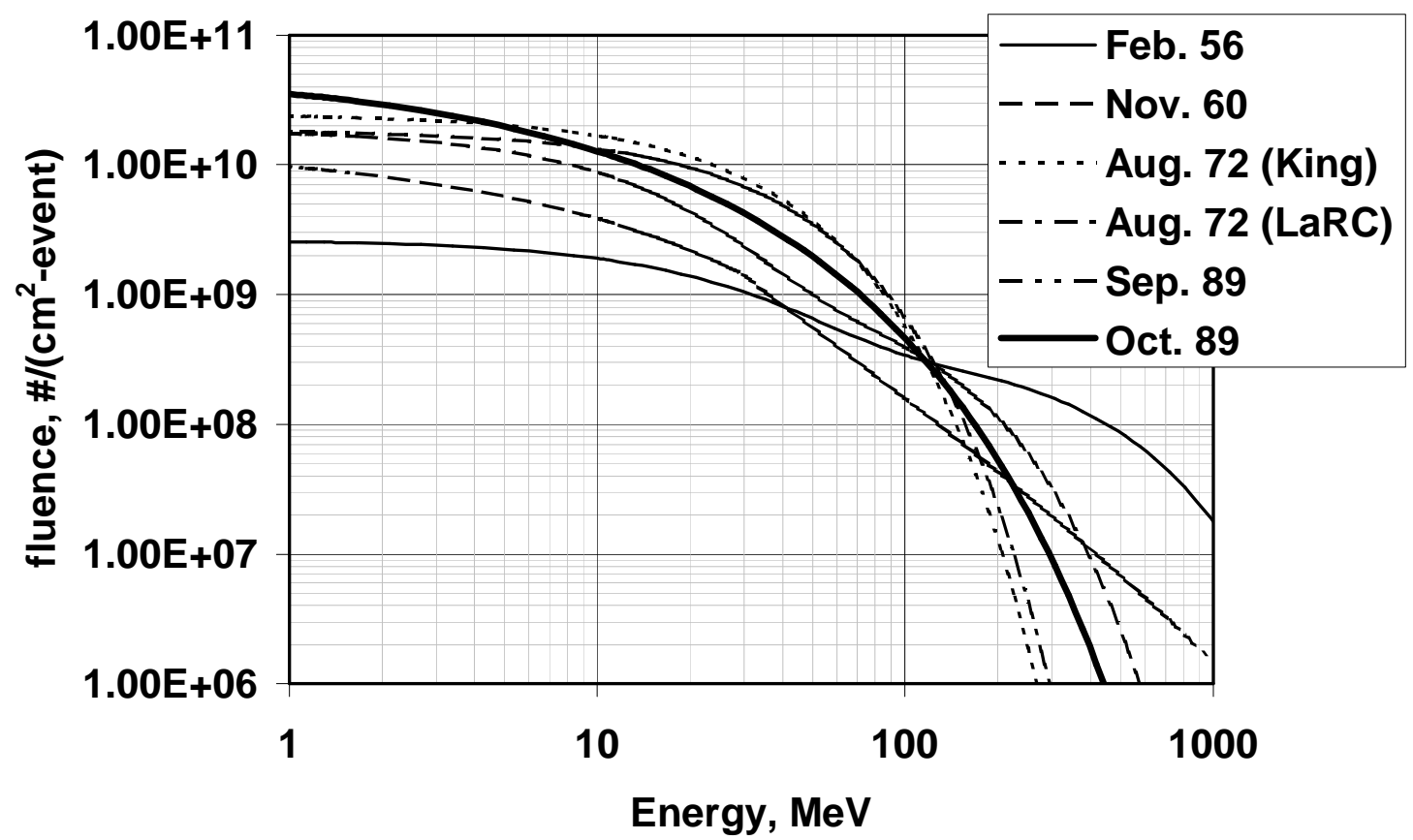

Figure 4b. Integral fluence spectra for six SPE events of cycle 19 and 20. 
Note that the limit of equation $10 \mathrm{~b}$, for a pure equilibrium spectrum (any particle type $\mathrm{j}$ ) at all energies is simply given by :

$$
\phi_{E}(E)=\frac{c}{S_{j}(E)}
$$

since the numerator in equation $10 \mathrm{~b}$ becomes a constant for $x>R(E)$. As an example, the equilibrium differential LET spectra for $\mathrm{c}=1$ is shown in Figure 5 below. Note that the equilibrium spectrum is seen in the high LET region for the stopping powers of hydrogen ions (mainly protons) and the helium ions (mainly alpha particles produced as nuclear decay products).

\section{Equilibrium d $\Phi(L) / d(L)$ vs. L spectra for 1977 GCR environment}

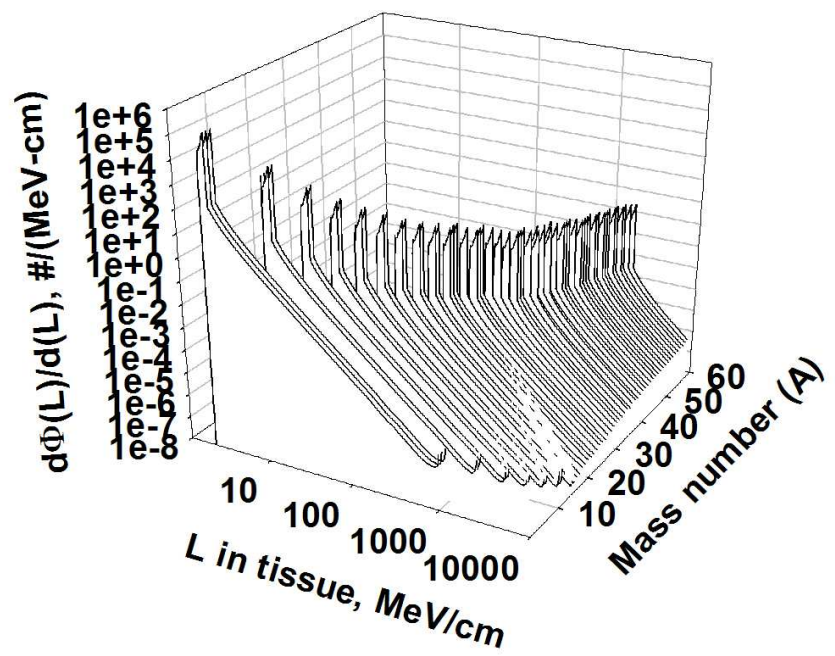

Figure 5. Equilibrium differential LET spectra for 1977 GCR solar minimum in tissue.

\section{INTEGRAL LET SPECTRA}

The integral LET spectrum is given as:

$$
\Phi(>L)=\int_{L}^{L_{\max }} \phi_{L}\left(L^{\prime}\right) d L^{\prime}
$$

which may be related to integral energy spectra as:

$$
\Phi(>L)=\Phi\left(>E_{1}\right)-\Phi\left(>E_{2}\right)+\Phi\left(>E_{3}\right)
$$

where $E_{1}, E_{2}$, and $E_{3}$ are the three roots (branch functions) of the relation $L=S(E)$.

The three branch functions of equation (15) are shown in Figure 6 for a range of $S(E)$ values for a number of ions and represent contiguous domains bounded by boundaries where $d L / d E=0$. 
The three branch functions for selected GCR ions in tissue

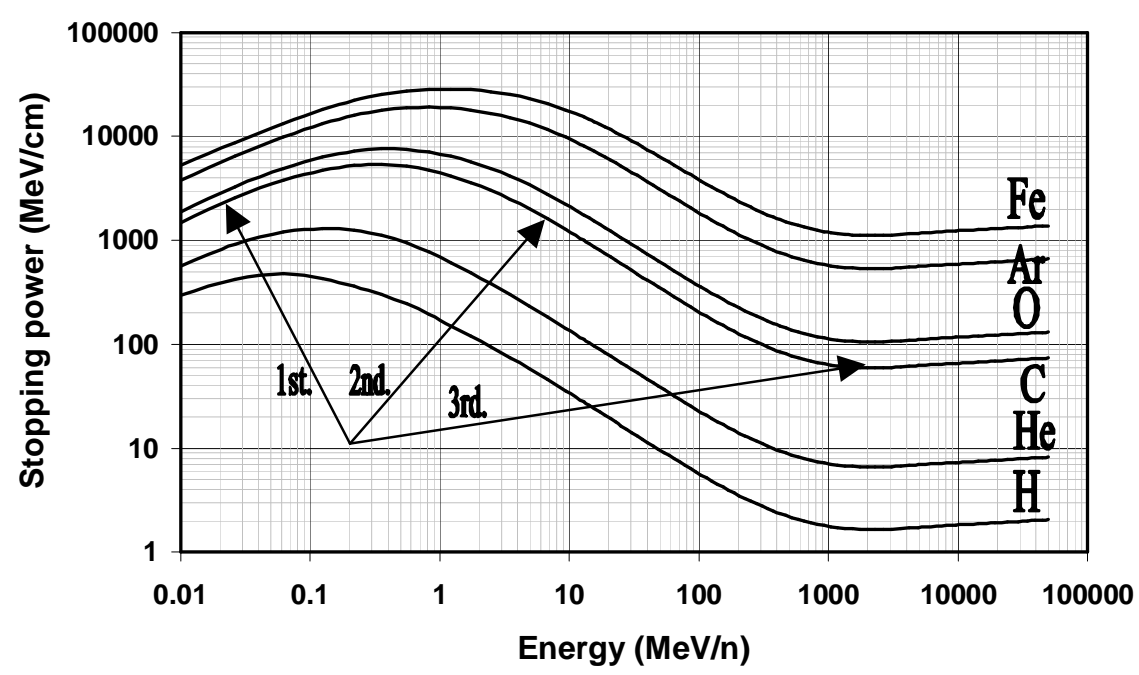

Figure 6. The three branch functions for selected GCR ions in tissue.

The integral LET spectra for the six SPEs discussed previously are shown in Figure 7. To gain perspective, we also evaluate the integral equilibrium spectral shape of equation (13) as:

$$
\Phi(>E)=\int_{E}^{\xi} \frac{d E^{\prime}}{L\left(E^{\prime}\right)}=R(\xi)-R(E)
$$

where we arbitrarily take the variable $\xi$ to be $10 \mathrm{GeV}$. The integral LET spectrum for $\xi>E_{3}$ is then:

$$
\Phi(>L)=R\left(E_{2}\right)-R\left(E_{1}\right)+R(\xi)-R\left(E_{3}\right)
$$

If we examine only the high-LET region, then:

$$
\Phi(>L)=R\left(E_{2}\right)-R\left(E_{1}\right)
$$

Results from equation (18) are shown in Figure 8. Thus, the integral LET spectra are characterized by the main branch of the LET curve as:

$$
\Phi(>L) \approx R\left(E_{2}\right)
$$

except at the highest LET values where

$$
\Phi(>L) \approx\left(E_{2}-E_{1}\right) / S\left(E_{\text {max }}\right)
$$




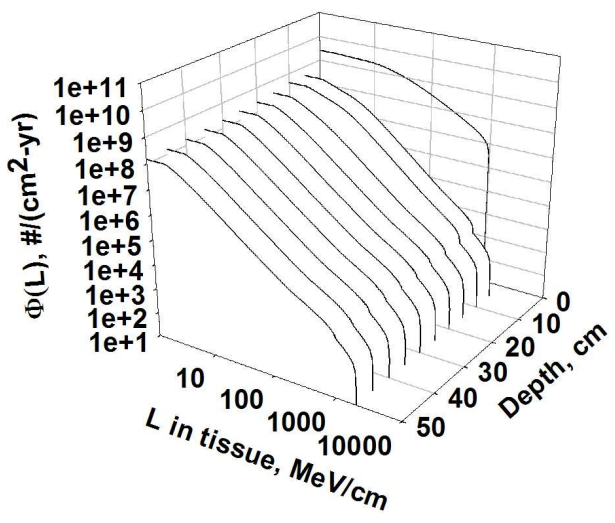

Freespace $\Phi(L)$ vs. L for Aug. 1972 SPE (King)

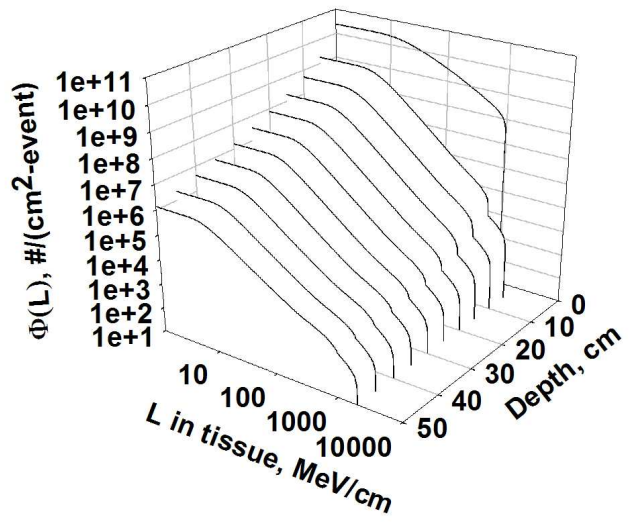

Freespace $\Phi(L)$ vs. L for Sep. 1989 SPE

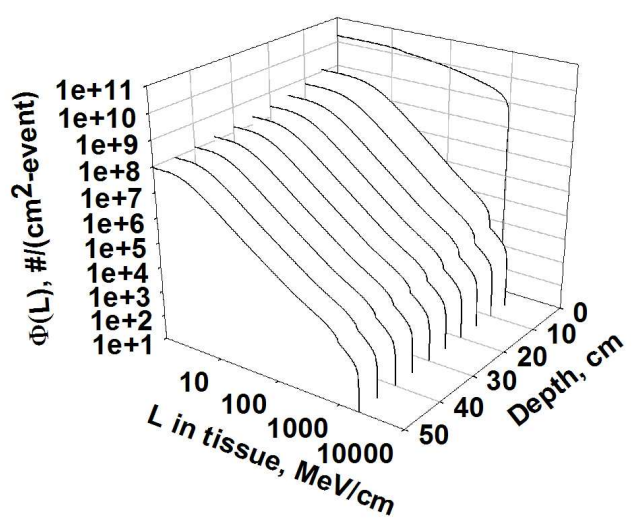

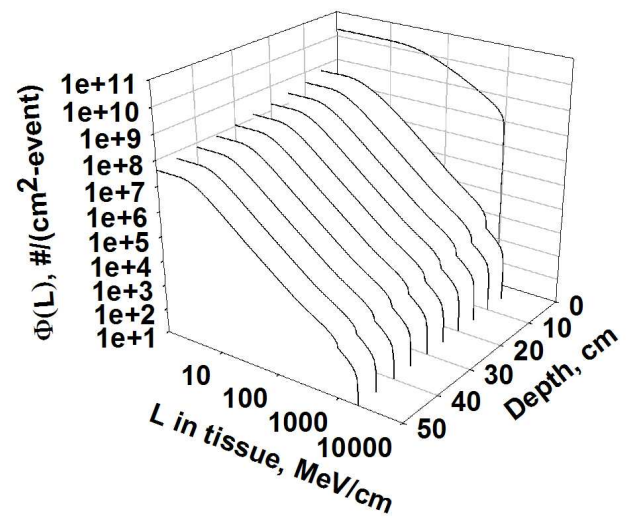

Freespace $\Phi(L)$ vs. L for Aug. 1972 SPE (LaRC)

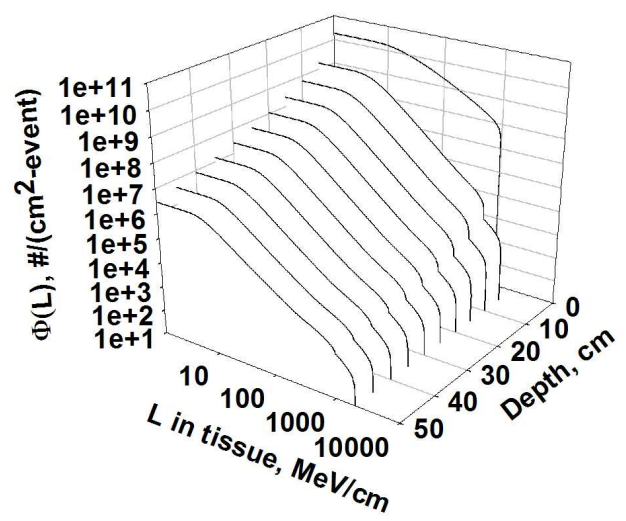

Freespace $\Phi(L)$ vs. L for Oct. 1989 SPE

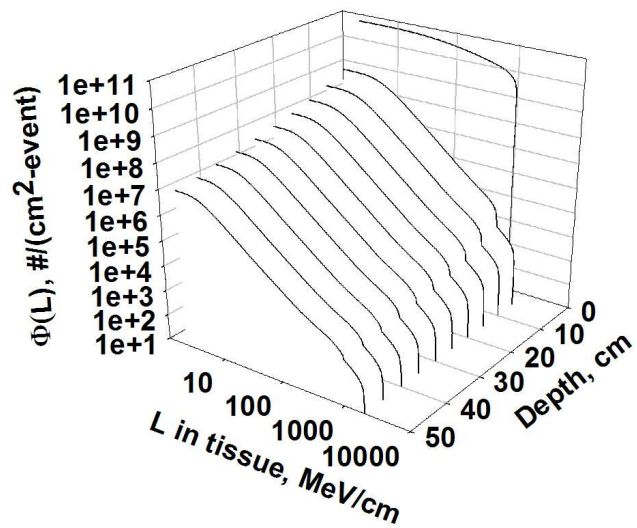

Figure 7. Integral LET spectra for six SPE events of cycles 19 and 20. 


\section{Equilibrium $\Phi(L)$ vs. L spectra for 1977 GCR environment}

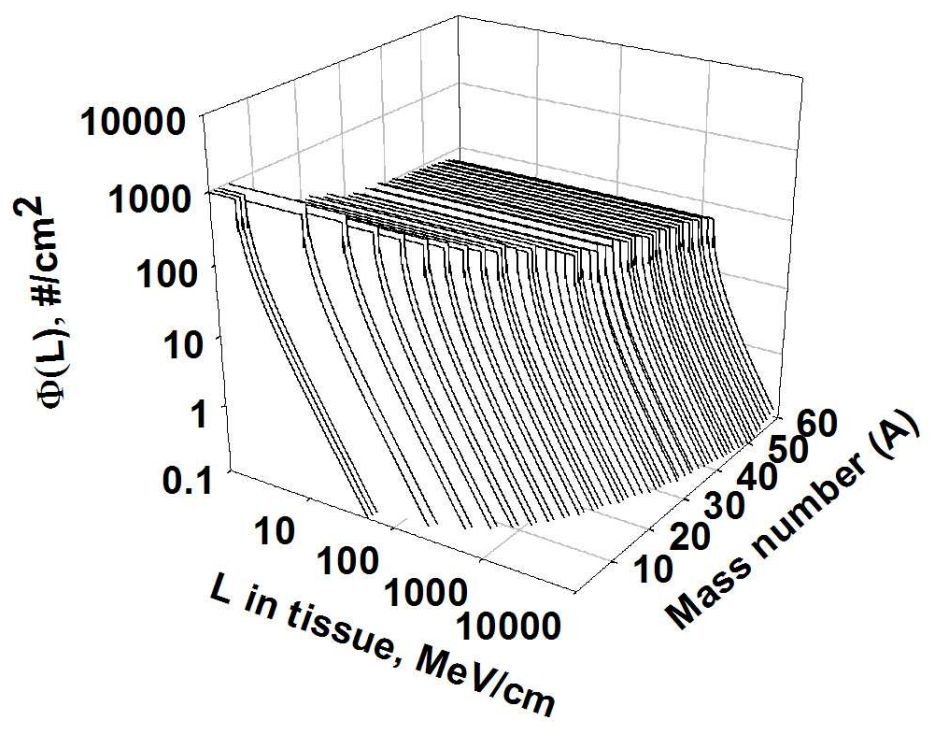

Figure 8. Equilibrium integral LET spectra for 1977 GCR solar minimum in tissue.

As a practical application of the numerical procedures described earlier, the differential LET spectra for the 1977 solar minimum GCR environment in free space and ISS trajectory are shown in Figure 9 at the tissue depth range of $0 \leq x \leq 50 \mathrm{~cm}$. As shown, the reduction of high LET components at larger tissue depths increases the lower LET component distributions. This is due to the fragmentation of the larger HZE components into smaller fragments. Note that there are two types of singularities: the first branch which is discontinuous at higher LET to the right of maximum ionization, and the third branch which is discontinuous to the left of minimum ionization. The corresponding integral LET spectra for 1977 GCR at free space and ISS trajectory are shown in Figure 10.

Freespace dФ(L)/d(L) vs. L for 1977 GCR environment

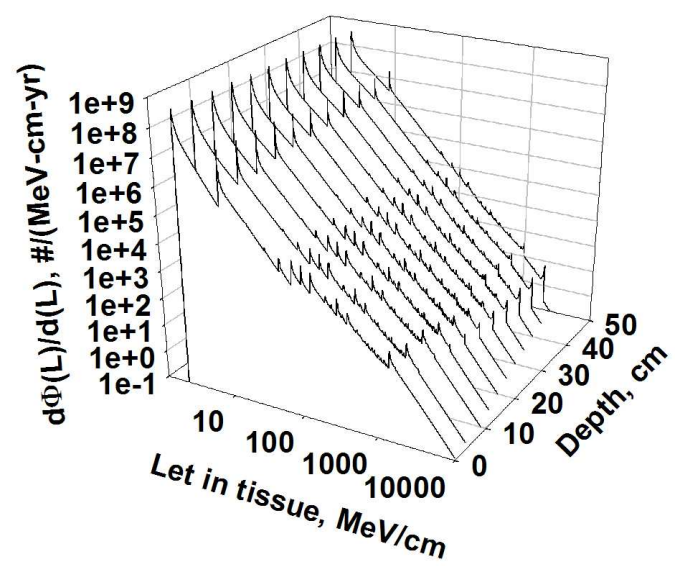

ISS dФ(L)/d(L) vs. L for 1977 GCR environment

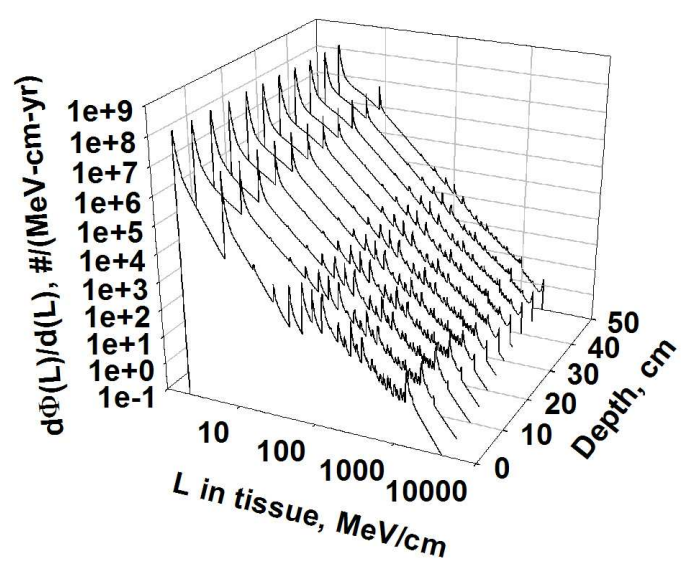

Figure 9. Differential LET spectra for 1977 GCR solar minimum in tissue. 

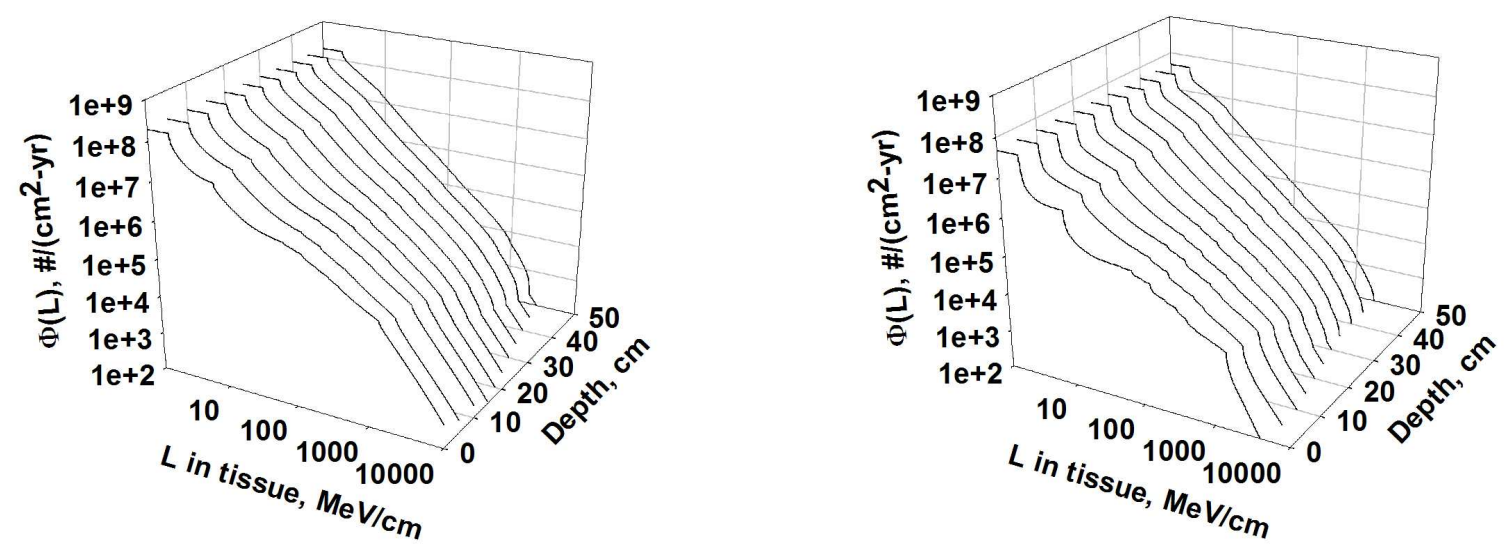

Figure 10. Integral LET spectra at 1977 GCR solar minimum in tissue.

\section{CONCLUDING REMARKS}

The problem of generating linear energy transfer (LET) spectra has been clearly delineated. Although integral LET spectra are continuous functions, the differential LET spectra are defined only over open sub-intervals and are unbounded near the open end points defined by minimum and maximum LET of specific ion types. An accurate method of constructing the spectra over their domain is provided. In future work, the numerical methodologies presented here will be used to analyze shielding applications for the protection of biological systems and electronic equipments.

\section{REFERENCES:}

1. Radiation Quantities and Units. ICRU Rep. 33, International Commission on Radiation Units and Measurements, c. 1980.

2. Hunter, A.: Dosimetric Response of Candidate Aliphatic Polymers as Shielding Materials to Space Ionizing Radiation, NASA Langley Research Center, LARSS Program (Aug 2005).

3. Wilson, J. W.; and Badavi, F. F.: A Study of the generation of Linear Energy Transfer Spectra for Space Radiations, NASA TM-4410, 1992.

4. Schaefer, H. J.: Exposure Hazards From Cosmic Radiation Beyond the Stratosphere and in Free Space. J. Aviation Med., vol. 23, no. 4, Aug. 1952, pp. 334-344.

5. The Quality Factor in Radiation Protection. ICRU Rep. 40, International Commission on Radiation Units and Measurements, Apr. 4, 1986.

6. Katz, R.; Ackerson, B.; Homayoonfar, M.; and Sharma, S. C.: Inactivation of Cells by Heavy Ion Bombardment. Radiat. Res., vol. 47, 1971, pp. 402-425.

7. Wilson, J. W.; Badavi F. F.; Cucinotta, F. A.; Shinn, J. L.; Badhwar, G. D.; Silberberg, R.; Tsao, C. H.; Townsend, L. W.; and Tripathi, R. K.: HZETRN: Description of a Free-Space Ion and Nucleon Transport and Shielding Computer Program. NASA Technical Paper 3495, May 1995.

8. Wilson, J. W.; Townsend, L. W.; Bidasaria, H. B.; Schimmerling, W.; Wong, M.; and Howard, J.: $20 \mathrm{Ne}$ Depth-Dose Relations in Water. Health Phys., vol. 46, no. 5, May 1984, pp. 1101-1111. 
9. Ziegler, J. F.: Helium Stopping Powers and Ranges in All Elemental Matter. Volume 4 of The Stopping and Ranges of Ions in Matter, J. F. Ziegler, organizer, Pergamon Press Inc., 1977.

10. Barkas, W. H.: Nuclear Research Emulsions. Volume 1-Techniques and Theory. Academic Press, 1973.

11. Wilson, J. W.: Analysis of the Theory of High-Energy Ion Transport. NASA TN D-8381, 1977.

12. Wilson, J. W.; Townsend, L.W.; Schimmerling, W.; Khandelwal, G. S.; Khan, F.; Nealy, J. E.; Cucinotta, F. A.; Simonsen, L. C.; Shinn, J. L.; and Norbury, J. W.: Transport Methods and Interactions for Space Radiations. NASA RP-1257, 1991. 


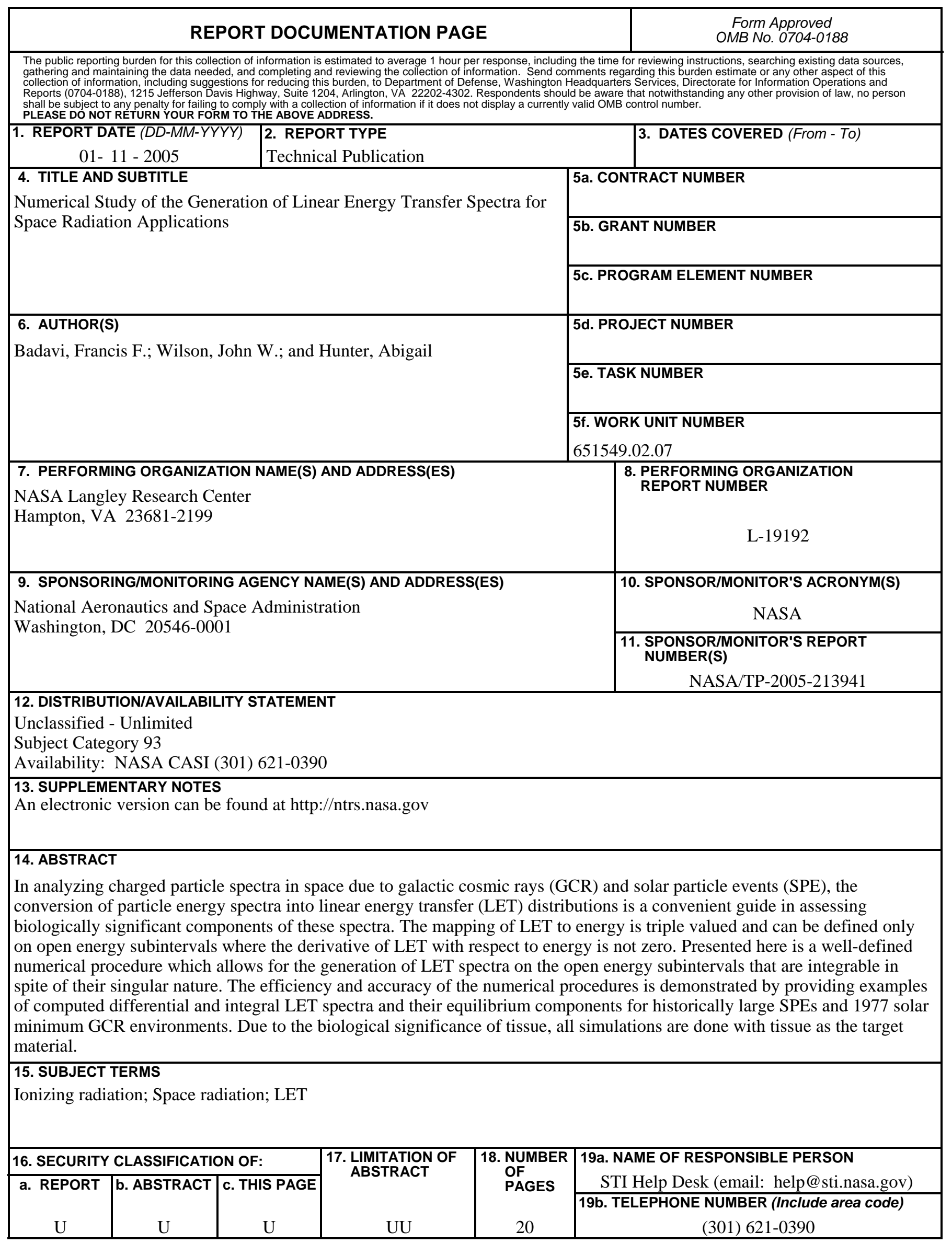

